\title{
DISPERSION COMPENSATION TECHNIQUES USED IN OPTICAL FIBER COMMUNICATION
}

\author{
Abha Jain, Anubhooti Jain, Bhavika Soni \\ E-Mail Id: abhajain005@gmail.com, anubhootijain0011@gmail.com, sonibhavika75@gmail.com \\ AravaliInstitute of Technical Studies, Udaipur, Rajasthan, India \\ Geetanjali Institute of Technical Studies Udaipur, Rajasthan, India
}

\begin{abstract}
Optical fiber is one of the most important communications media in communication system. Due to its versatile advantages and negligible transmission loss it is used in high speed data transmission. In order to maintain high value of optical signal to noise ratio (OSNR) for having a signal of good quality it is important to compensate dispersion in optical finer communication system. With Dispersion optical fiber's performance degrades. Research was carried on how dispersion can be compensated. Dispersion compensation in fiber optical communication system has become a topic of great importance and research these days because any presence of dispersion might lead to pulse spreading which might cause inter symbolic interference (ISI) which leads to signal degradation. In this manuscript, various types of dispersions are discussed in a brief way. Also different methods of dispersion compensation like-Dispersion compensation fiber (DCF), Electronic Equalizer, Fiber Bragg Grating (FBG) and digital filters are discussed in detail.
\end{abstract}

Keywords: Optical Transmission System, Fiber Bragg Grating (FBG), dispersion compensation, Opt system simulator, parameters.

\section{INTRODUCTION}

In Fiber Optic Communication system we transmit information from one point to another inform of light signals or pulses [1,2]. The light pulses are modulated by a electromagnetic wave which acts as a carrier signal. It is the large bandwidth that can be achieved by using optical fiber communication that lead to a worldwide development and installation of fiber optic links. The performance of an optical fiber communication link in degraded by many factors such as scattering, bending losses, absorption, scintillation, nonlinear effects and chromatic dispersion. In single mode fibers[3], intermodal dispersion is absent simply because energy of the injected pulse is transported by a single mode. However, Pulse broadening does not disappear altogether. The Group velocity associated with the fundamental mode is frequency dependent because of chromatic dispersion. Different spectral components of the pulse travel at slightly different group velocities, knows as group- velocity dispersion (GVD), intarmodal dispersion.

Discussions on these topics will tell about how GVD limits the light wave systems having single mode fibers. Mechanism that causes dispersion in a single mode fiber is chromatic dispersion and Polarization-mode dispersion (PMD). Intramodal dispersion has two contributions, material dispersion and wave guide dispersion. In single mode fiber where intermodal dispersion does not exist and material dispersion is very small.

\section{DISPERSION}

Dispersion is characterized as pulse spreading in an optical fiber. As a pulse of light spreads through a fiber, components, for example, numerical aperture, core diameter, refractive index profile, wavelength, and laser line width make the pulse widen. Dispersion increments along the fiber length.

The general impact of dispersion on the performance of a fiber optic framework is known as Inter symbol Interference (ISI). Inter symbol interference happens when the pulse spreading caused by scattering causes the output pulse of a framework to overlap, rendering them undetectable. Dispersion is by and large partitioned into three classifications: modal dispersion, chromatic dispersion and polarization mode dispersion.

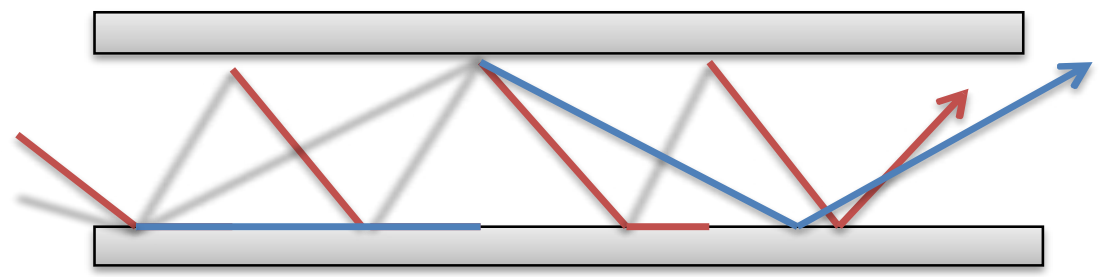

Fig. 2.1 Modal dispersion

\subsection{Modal Dispersion}

It causes pulses to spread out as they travel along the fiber,the more modes the fiber transmits, the more pulses spread out. The arrival of different of the light at different times is called Modal dispersion.

\subsection{Chromaticdispersion(CD)}

It is associated with wavelength dependent pulse spreading due to the fact that different wavelengths of lightpropagate at slightly different velocities through the fiber because the index of refraction of glass fiber is awavelength-dependent quantity; different wavelengths propagate at different velocities.Chromatic Dispersion (CD) that causes pulse broadening depending on wavelength. 


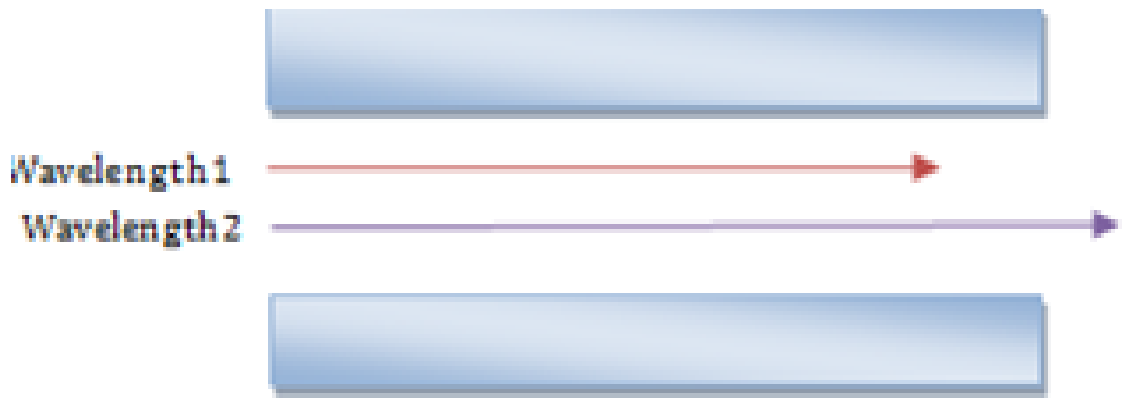

Fig. 2.2 Chromatic Dispersion

Chromatic dispersion consists of two parts: material dispersion and waveguidedispersion

\subsection{Material Dispersion}

It is caused when a light travels within a medium whose refractive index is wavelength dependent, the light will experience material dispersion regardless of whether the medium is enclosed or not.As it depends on the material and can't be changed.

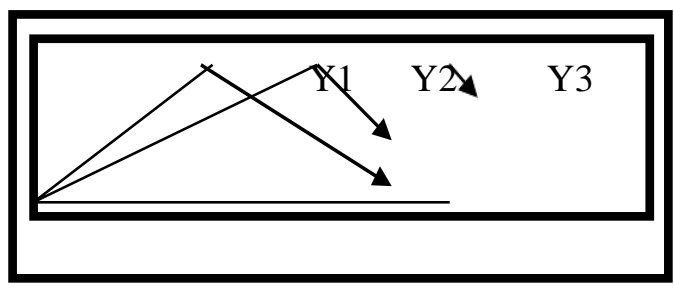

Fig. 2.3 Material Dispersion

\subsection{Wave guide dispersion}

It occurs when light travels with in an enclosed medium. Waveguide dispersion depend on the refractive index profile and can be changed. It can be used to produce dispersion-decreasing fibers in which GVD decreases along the fiber length.

\section{NEED FOR DISPERSIONCOMPENSATION}

11Due to the dependence of speed of information carrying signal on the refractive index of the fiber which depends on the wavelength of the signal carrying information, different signals having different wavelengths reach the output of the fiber at different times as in case of a multi-mode fiber. Evenin a single mode fiber the information carrying signal does not consists of a single wavelength rather a continuous group of wavelengths called the spectral with of transmitting source. These wavelengths experience different refractive index and hence travel with different velocities and reach output of the fiber at different time causing the pulse to spread [4]. Now if the data rate of the information signal is increased, the pulses at the output may overlap with each other as shown in fig. 3.1. These causes inter symbolic interference (ISI). Due to inter symbolic interference we cannot increase the data rate of the fiber optic communication link beyond a certain limit. As a result dispersion is a limiting factor on data rate of fiber optic communication link. Thus in order to achieve high data rates, dispersion compensation is the most important requirement in fiber optic communication link.

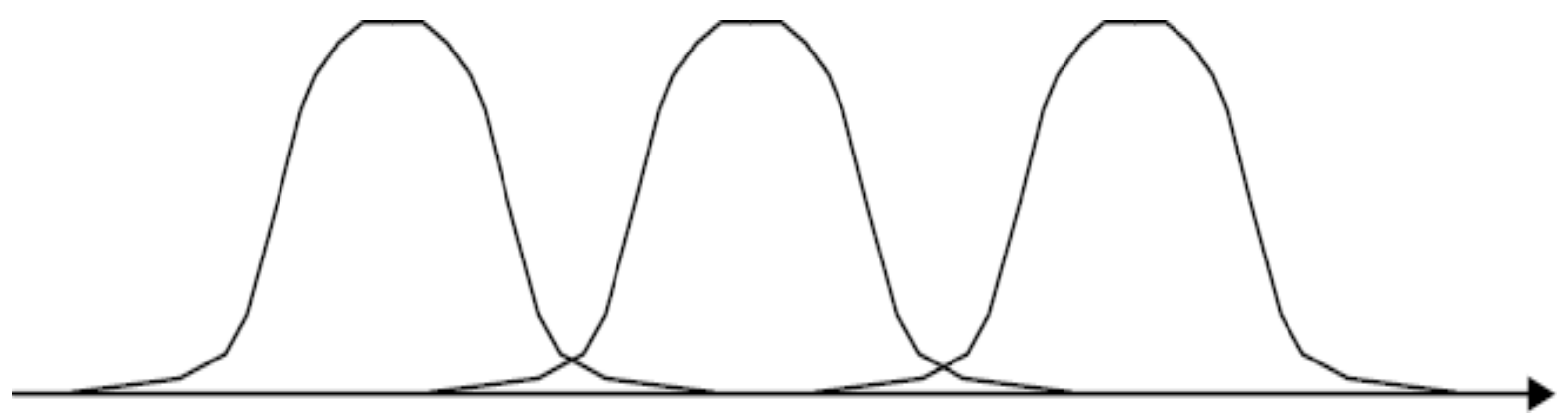

Fig. 3.1 Inter symbolic Interference due to Dispersion

\section{DISPERSION COMPENSATION TECHNIQUES}

\subsection{DCF(Dispersion Compensation Fiber)}

Too much dispersion in a system leads to power penalty and poor quality of service. Users want more bandwidth and dispersion limits bandwidth in optical fibers.

In Chromatic dispersion[5], it gives an opportunity to compensate for dispersion along the entire span of the fiberoptic link. In DCF the positive dispersion can be compensated by inserting a piece of fiber with a negative dispersion characteristic so that the total dispersion of the link will be almost zero.

DOI Number: https://doi.org/10.30780/specialissue-SCRDSI-2021/004

pg. 14 
SCRDSI-2021, Aravali Institute of Technical Studies, Udaipur (Rajasthan), India

International Journal of Technical Research \& Science (Special Issue) ISSN No.:2454-2024 (online)

DCF compensation needs very high negative dispersion coefficient with DCF's to compensate dispersion in a narrow band frequency.

There are three schemes in Dispersion Compensation Fiber

\subsubsection{Precompensation}

In Pre compensation[6] DCF is placed before SMF. The designing consists of DCF,EDFA and SMF. Purpose of EDFA after DCF is that it provides periodic amplification. Dispersion Parameter is expressed in $\mathrm{ps} / \mathrm{nm} / \mathrm{km}$ and is in negative.

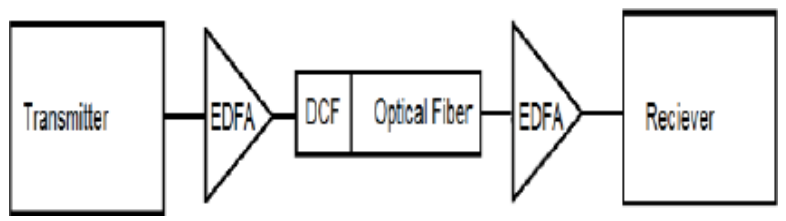

Fig. 4.1 Pre Compensation

\subsubsection{Post Compensation}

As dispersion causes pulse broadening and pulse distortion. Compensation is post compensation in which $\mathrm{SMF}$ (single mode fiber) is placed beforeDCF.

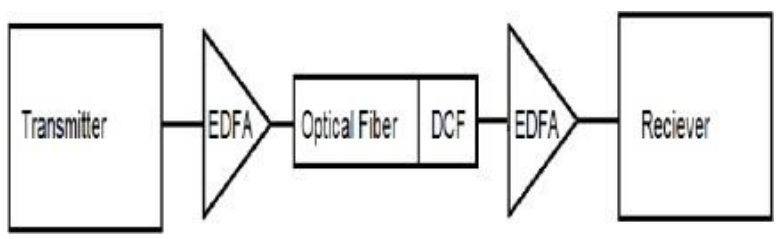

Fig. 4.2 Post Compensation

\subsubsection{Symmetric Compensation}

In symmetric compensation, DCF is placed before and after the standard fiber.

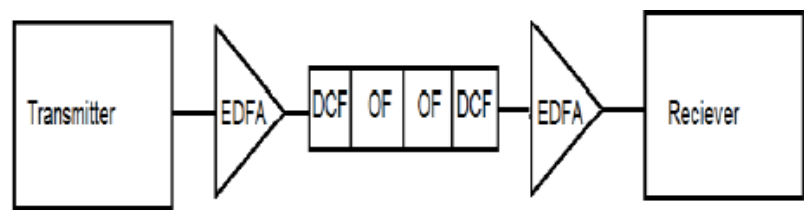

Fig. 4.3 Symmetric Compensation

\subsection{Fiber Bragg Grating (FBG)}

The idea of Fiber Bragg Grating was initially presented in 1980andhasbeenutilizedasapartofafewapplicationsand generally inquired about. It comprises of a direct intelligent gadget whose intelligent record profile changes straightly concerning length of the fiber. The grinding mirrors the light contingent on the wavelength of the light entered in the grinding [7]. The light with bigger wavelength ventures a more prominent separation in the grinding before getting considered the other hand beam with littler wavelength voyages a shorter sparation in side the grinding before getting reflected. Thus, the beam which is extended by the chromatic passing so as to scatina SMF is packed through a Fiber Bragg Grating.

FBG is used in two configurations, Precompensation it defines when the FBG is placed at the starting of optical link and before amplifier. Following figure describes the concept of pre compensation.

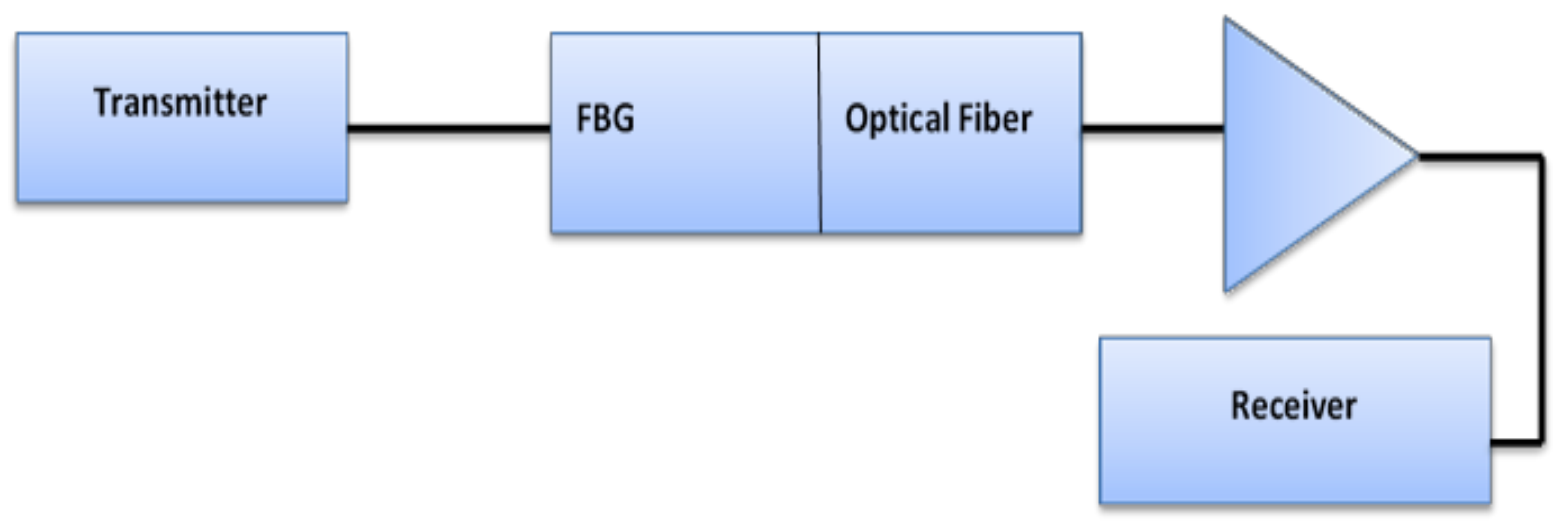

Fig. 4.4 Pre Compensation

\subsection{Post Compensation}

It defines the situation when FBG s placed at the end of optical link. Following figure describes the post compensation.

DOI Number: https://doi.org/10.30780/specialissue-SCRDSI-2021/004

pg. 15 


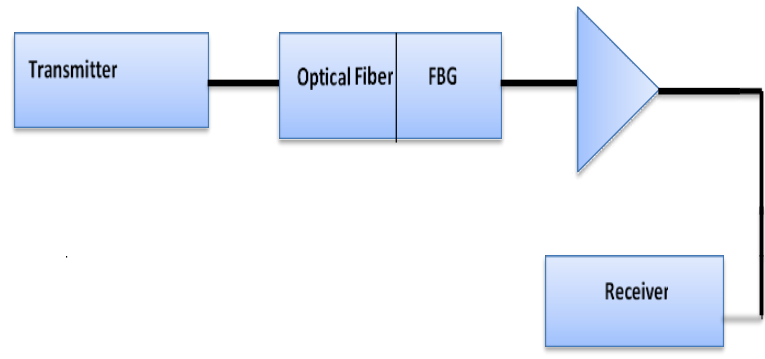

Fig. 4.5 Post Compensation

\subsection{Erbium-Doped Fiber Amplifier (EDFA)}

Erbium-Doped Fiber Amplifier (EDFA) is an optical amplifier used in the C-band and L-band, where loss of telecom optical fibers becomes lowest in the entire optical telecommunication wavelength bands. To face chromatic dispersion, we use Fiber Bragg Grating. EDFA is used as a amplifier in the link as it can operate in C band as well as in L band and boost the system performance. As insertion loss is less in FBG and it also helps in reducing cost of the system. On the other hand DCF increases total losses and cost of the system.

\section{CONCLUSION}

There are many techniques that can be utilized to compensate dispersion in an optical fiber communication link. Dispersion compensating fibers are considered to be the simplest as they are used in the fiber optical loop along with the standard fiber and posses opposite dispersion which is used to mitigate dispersion. But the insertion loss for a DCF is very high. Finer Bragg Grating is a very compact device with low insertion loss and compensates dispersion by compressing the pulse which passes through it.

\section{REFERENCE}

[1] C.H. Cheng, "Signal Processing for Optical Communication," IEEE Signal Processing Magazine, vol. 23, no. 1, pp. 88-96, 2006.

[2] C.D. Poole, J.M. Wiesenfeld, D.J. DiGiovanni, A.M. Vengsarkar, Optical fiber-based dispersion compensation using higher order modes near cutoff," Journal of Lightwave Technology, vol. 12, no. 10, pp. 1746-1758, 1994.

[3] M. I. Hayee and A. E. Willner, "Pre- and post- compensation of dispersion and non linearities in 10-Gb/s WDM systems", IEEE Photon. Tech. Lett. 9, pp. 1271,1997.

[4] R.I. Killey, P.M. Watts, M. Glick, and P. Bayvel,

[5] "Electronic dispersion Compensation by signal pre-distortion," Optical Networks Group, Department of Electronic and electrical Engineering, University College London, Torrington Place, 2006

[6] Maninder singh, Maninder Lal singh "A novelalgorithm to integrate synchronousdigitalhierarchy networks into Optical Transport Network using mixed line rates"2014.

[7] G.P. Agrawal, Fibre Optic Communication system,3 rdedition, WilleyIntersience,2002.

[8] G. Lenz and C.K. Madsen, "General Optical All-Pass Filter Structures for Dispersion Control in WDM Systems,” Journal of Lightwave Technology, vol. 17, no. 7, pp. 1248- 1254, 1999. 